tightly, and will withstand any reasonable strain necessary for passive movements at an early stage of the after-treatment. It has been shown over and over again in arthrectomies operated on by Volkmann's plan of transverse section of the patella that this bone unites rapidly and firmly by bony material when perfectly coapted, and the present cases demonstrate the same fact.

Three objections may be urged to the procedure now described. First, that the suture may give trouble in the joint, inasmuch as it lies in the axis of movement. However, we need not expect this, and, as a matter of fact, none of the four patients treated by it are conscious of the presence of the suture in the joint. Next the knot might possibly cause pain in front of the knee, but this has not been the case with any of these patients. Lastly, the joint has to be opened and transversed by a needle. But in answer to this objection it may be urged that this is no more than what is done in aspiration, which has been long recognised as a perfectly safe procedure in the hands of careful surgeons.

From the numerous efforts that have been made of late years to perfect the methods of suture of the patella, and the fact that so far none of them has come into general use, it may, I think, be safely predicted that surgeons will not rest until at last some simple plan of operation is devised which will ensure perfect coaptation of the fragments in recent fractures with little or no risk $(a)$ as to infection of the joint, (b) as to subsequent separation of the fragments, (c) as to residual stiffness. This procedure, whatever it be, must be so simple as to require no extraordinary skill on the part of the surgeon. It must be so safe as to justify its being used at once for all recent fractures without hesitation. It must give such good results as to function as to outdo everything that has been achieved through treatment by splints.

Whether the method I have here described is a step in this direction or not time only can slow. But having myself operated by the open method on other cases, and so far with successes and no failures, and having seen other methods employed by my colleagues, I am in a position to contrast several of the plans in use, and can say that, were I to fracture my own knee-pan, I should prefer to have it treated by the permanent subcutaneous suture rather than any other.

I have hesitated to refer to this procedure as a new one; it may have been employed by others without my having been able to discover the fact after careful search and inquiry. But the question is not whether it is novel but whether it is good, and if other surgeons have ever employed it I shall be greatly interested to know whether they have been as favourably impressed by it as $I$ have in the results of these four cases.

I have just examined a fifth case in which I operated a week ago (February 2nd) by the same method. The joint is quite quiet and I have already commenced passive movement. The whole procedure only lasted four and a-half minutes.

\section{CONTRIBUTIONS TO THE PHYSIOLOGY AND PATHOLOGY OF THE MAMMALIAN HEART.}

Bx C. S. ROY, F.R.S., M.D., Professor of Pathology, University of Cambridge : AND

J. G. ADAMI, M.A., M.B.,

Fellow of Jesus College, Cambridge.

(An Abstract).

[From the Cambridge Pathological Laboratory.]

We have sought to study the action of the mammalian heart in conditions (unexcised and intact) as nearly approaching the normal as we were able to make compatible with the employment of exact methods of research.

In our complete paper, which will shortly be published, we describe a cardiometer which we,employed to measure the "contraction volume" and the "output,", ${ }_{2}$ as well as the

${ }^{1}$ Communicated to the Royal Society on February 11th, and printed by kind permission of the President and Council.

2 "Contraction volume" is the volume of blood expelled by the heart at exch contraction. "Output" is the volume of blood expelled by the heart iu any given time. changes in the volume of the heart other than those due to its rhythmic contractions and expansions. A description will also be given of the method of employing it, together with a statement as to the degree of the accuracy with which, according to our experience, the instrument supplies information regarding changes in the volume of the heart. We will also describe an automatic "counter," which we employed for measuring out and recording the output of the heart, as obtained by the cardiometer.

Another instrument which was found of great value in our work is our myocardiograph, which we made use of to record the contractions and expansions of any part or parts of the ventricular or auricular walls without interfering with the mcvements of the heart. In most cases we employed this instrument to obtain simultaneous records of the contractions of one auricle and one ventricle. We have great doubts as to the value of observations made on the heart by "button" cardiographs.

Each ventricle is sufficiently nearly spherical to permit us to deal with the mechanics of the ventricles on that basis, and, whatever the method of research employed, it is important to keep in mind the relationship of the circumferences of spheres and their cubic contents. If the circumferences of spheres be taken as the abscissæ, and the cubic contents represented as ordinates, the curve obtained is a " cubical parabola." For example, reduction by 1 inch of the circumference of a sphere of 5 inches circumference is accompanied by a reduction of the contents equal in amount to that which accompanies the lessening by $\frac{1}{4}$ inch of a sphere of 10 inches circumference, although in the one sphere the circumference is lowered $\frac{b}{b}$, and in the other $\frac{1}{40}$.

It is important, also, to keep in mind that the strain on the walls of a hollow spherical shell increases uniformly with the circumference ; in other words, that the resistance to contraction of the ventricular walls becomes greater as the circumference is increased. It is well, also, to note that a change of pressure in the aorta or pulmonary artery has the same effect on the resistance to contraction offered to the left or right ventricular walls as an increase or decrease of a weight hung on a parallel-fibred voluntary muscle which is caused to contract by single induction shocks causing maximal contractions. If you increase the weight, it is not pulled up so high, and vice versa. An analogous result takes place in the case of the ventricles. When, from any cause, the resistance to contraction is increased, or their force of contraction, from any cause, is reduced, their walls contract less completely, and the amount of "residual "blood leit in their cavity at the end of systole is increased.

We now come to the effects of the vagus nerve upon the heart. We begin with the changes in the "contraction volume," and find that, at first sight, our curves seem to show that, other things being equal, the volume of blood expelled at each systole varies in inverse ratio to the rapidity of heart beat. We find, however, that this general law does not hold good for vagus slowing (if, indeed, it be exact for slowing of any kind), which is found to be accompanied by a lowering of the "output." That, with moderate slowing, this diminution of the output may be as much as 30 or 35 per cent.

The increase in the amount of residual blood in the heart, which is produced by vagus excitation, does not necessarily indicate any weakening of the ventricular contractions.

We next analyse myocardiographic records of the action of the vagus upon the heart, and find that the auricular contractions are weakened or arrested, noting that the influence of the vagus upon the force of the auricular contractions bears no constant proportion to the vagus slowing. By fairly strong vagus excitation or by muscarin the auricles may be completely arrested, it may be, for hours. This complete arrest is, in some cases, led up to by progressive weakening, but sometimes arrest occurs immediately after fairly strong beats, or with fairly strong beats presenting themselves at times during the arrest. These latter cases may be explained by weakening of the excitations which reach the auricles from the sinus, although they are possibly due to diminished excitability of the auricles.

On coming to the effect of the vagi upon the ventricles, we find that the distension of the heart during vagus actions is due to the ventricles being more expanded, both in diastole and in systole. We point out that the increased volume of 
the heart at the end of systole is a necessary result of the increase in the ventricular contents, and combat the conclusions of those who ascribe it to weakening of the ventricular contractions, pointing out that the greatly increased contraction volume increases to a corresponding extent the work done at each contraction. We give detailed reasons for concluding that this suffices to explain the apparent diminution in the amplitude of the ventricular contractions.

We then examine the influence of the vagus upon the tonus of the relaxed ventricles, and point out that the great distension during vagus action is due entirely to increased intraventricular pressure during diastole, and not, as has been asserted by some. to any change in the elasticity of the relaxed ventricular wall.

Next we consider the cause of the rise of venous (systemic and pulmonary) pressure, and find that this is due, not to any increase in the amount of blood entering the veins in a given time or to contraction of their walls, but that it is to be ascribed to the diminished inflow into the ventricles.

The cause of this diminished inflow into the ventricles leading to corresponding diminution of the output is twofold, namely, weakening or arrest of the auricles, and, secondly, the elastic resistance of the ventricular wall to distension. We show that this explanation must apply to both sides of the heart, and that observed facts correspond with it.

We then consider the after-effects of vagus excitation, and show that the temporary increase in the output which is sometimes present, may be explained by a temporary increase in the force of the auricular contractions, and by the venous pressure taking some little time to fall after the vagus excitation has ceased.

After this we examine the influence of the vagus upon the heart rhythm, and show that, when the vagus excitation reaches a certain degree (varying in different animals), the ventricles begin to beat independently of the sinus and auricles; that this rhythm, which is at first slow and irregular, gradually becomes fairly rapid and almost completely regular. This rhythm, we show, must be looked upon as the same as that which, as Wooldridge and Tigerstedt observed, makes its appearance when the ventricles are severed from the auricles. We point out, however, that the independent ventricular rhythm of vagus action is characterised by the slowness with which it establishes itself. This characteristic is due to the lowering of the excitability of the ventricles produced by vagus action, and we adduce a considerable number of facts showing that the vagus does lower the excitability of the ventricles, and that, by means of muscarin and by discontinuous stimulation of the vagus, it is possible to isolate the influence of the vagus on the rhythm and force of the auricles from its influence upon the excitability of the ventricles. The power of the vagus to stop the ventricles temporarily can only be explained by this diminution of their excitability.

We show that, with a certain degree of vagus excitation, irregularity of the ventricles necessarily results in consequence of the sinus and the idio-ventricular rhythms interfering with one another; that this is the common cause of irregularity; and that irregularity may also he ca 1sed by the auricles not responding to all the impulses which reach them from the sinus.

We explain that, in rare instances, direct excitation of the vagus may so lower the excitability of the ventricle that the contractions may not extend over the whole of their walls, and may in this way produce the apparent reakening which is sometimes met with.

We then pass on to study the effect of direct excitation of the nervi augmentores (accelerantes) upon the heart, and find that the acceleration of the rhythm may be extremely slight if the heart be beating fast, and that the acceleration and augmentation of force of the heart bear no constant proportion to one another. The augmentor nerves increase the diastolic expansion of the auricles and also increase their systolic contraction, but these two effects do not go hand-in-hand.

Excitation of the augmentors increases the output of the heart, owing to the increased force and frequence of the auricular contractions, the result of this being that the pressures in the systemic and pulmonary arteries rise. while the systemic and pulmonary venous pressures fall. If there be but little quickening, the contraction volume of the ventricles is increased.
The augmentors, on direct stimulation, cause a slight increase in the diastolic expansion of the ventricles, which is passive in nature and due to the increased force of the auricular contraction. The force of the ventricular contractions is increased; they contract more completely, diminishing the amount of residual blood, in spite of the fact that the arterial pressure is usually somewhat raised.

There are certain nerve fibres other than the nervi augmentores proper which pass from the stellate ganglion to the heart, sometimes by the annulus of Vieussens to the inferior cervical ganglion, but sometimes as separate branches passing directly to the beart from the ganglion stellatum or the annulus. On peripheral excitation of the cut nerves there is marked weakening of the contractions both of the auricles and of the ventricles, usually with some degree of slowing, this being sometimes followed on cessation of the excitation by a very well-marked increase in the force and frequence of the auricular and ventricular contractions. They may be vaso-constrictors for the coronary vessels, although we can, in the meantime, give no proof of this.

There are nerve fibres which descend to the heart by the vago-sympathetics, which, on excitation under certain conditions, increase the force and frequence of beat of the auricles and ventricles, and which may be vaso-dilators for the coronary vessels.

Reflex excitation of the vagus produces results which are the same as those, above described, of direct excitation of the nerve, the curves being, however, more typical and satisfactory than those obtained on peripheral excitation of the nerves.

Excitation of a mixed nerve like the sciatic usually produces effects on the heart similar in kind to those caused by direct excitation of the augmentors, but the phenomena are complicated by the greater rise of the pressure in the systemic arteries. Sometimes the increase in force of the left ventricle more than counterbalances this increased resistance to contraction, and the amount of residual blood in the left ventricle is reduced; in other cases the increase in force of the ventricular contractions is not sufficient to counterbalance the increased resistance, and the residual blood in the left ventricle is increased.

We find that excitation of the central end of a mixed nerve, like the sciatic or splanchnic, often affects both the augmentor and vagus centres in the medulla, and that, in nearly all such cases, ti.e augmentor centre is the more strongly excited of the two, so that augmentor effects show themselves during the excitation, but are succeeded by vagus action on ceasing to excite the nerve. In many cases augmentor effects alone show themselves. When excited reflexly the augmentor centre begins and ceases to act earlier than the vagus; the opposite, therefore, to what takes place with direct excitation. In rare cases the excitation of the vagus centre may be stronger than that of the augmentor from the first. Although, in the absence of any augmentor action, the vagus does not reduce the force of the ventricular systole, it does unmistakahly have the power of inhibiting the strengthening influence which the augmentors exert upon the ventricular contractions.

On examination of the part played by the vagus in the economy, we find that vagus excitation relieves the heart of work and therefore of tissue waste to as great an extent as is compatible with a continuation of the circulation, and conclude that the vagus acts as a protective nerve to the heart, reducing the work thrown upon that organ when, from fatigue or other cause, such relief is required by it. The presence of fibres in the sciatic and other mixed nerves which can cause reflex excitation of the vagus would seem to indicate that this nerve may be used by other parts of the body to diminish the output of the heart and lower the blood pressure, thereby reducing the activity of the circulation as a whole. The influence of the blood pressure in the systemic arteries on the degree of vagus activity and the readiness with which the vagus centre is called into play on raising the intracranial pressure indicate that the vagus mechanism is specially employed in lowering the circulation so as to limit cerebral congestion. The vagus acts chiefly in the interests of the heart and central nervous system.

The power of the vagus over the heart is limited, and the idio-ventricular mechanism, which comes into play when the vagus action exceeds a certain limit, must be looked upon as 
the means by which arrest of the circulation and death is prt vented, whenever from any cause the nerve exerts a maximrm influence. The power of the vagus to lower the excitabijity of the ventricles, makes their temporary arrest possible, but this reduction of the excitability of the ventricles cannot be kept up, no matter how strong the stimuli applied to the nerve, for a period long enough to endanger the economy.

We find that the function of the augmentor nerves in the economy is to increase the work and tissue-waste of the heart, as part of the mechanism by which the nervous system governs the circulation, and that the augmentor mechanism sacrifices the heart, so to speak, in order to increase the output of the organ and enable the ventricles to pump out their contents against a heightened arterial pressure. Such excessive action of the heart is limited by the vagus, .which, as we have seen, readily steps in so soon as the call for an increased supply has ceased. It may do so earlier, presumably because the increased blood pressure or the fatigue of the heart calls for vagus intervention.

We then consider the mode of interaction of the vagi and augmentores, and point out that when the vagi are paralysed by section or atropine the augmentores would seem to have no control over the cardiac rhythm, and that, therefore, they can only act by inhibiting the influence of the vagi on therhythmic centre of the heart. When neither nerve is acting on the auricles, these latter contract with a certain force, which is increased by the augmentores and diminished or inhibited by the vagi. The force of the ventricular contractions is increased by augmentor action; this increase can be inhibited by vagus excitation, which latter has otherwise no power to reduce the strength of ventricular contractions.

The force of the heart's contractions is influenced by other factors than the vagi, augmentores, and other nerves. The pressure of the blood in the coronary arteries is one of the most important of these factors. If this be lowered the contractions of both auricles and ventricles diminish in strength, while a rise of pressure in the systemic arteries causes an increase in the force of the heart's contractions, so that the force of the heart's contractions is to a certain extent regulated automatically by changes in the blood pressure in the aorta, which is one of the variable quantities affecting the work of the left ventricle.

Change of the volume of blood in the body affects greatly the contraction volume and output of the heart. Injections into the veins of a volume of defibrinated. blood equal to onetenth of the total amount of blood in the body may double the output. It is important to note here that there is no increase in the strength of the ventricular contractions; increase in the work, therefore, of the ventricles due to increase in the output has no tendency to automatically increase the force of the ventricular contractions, as is the case with rise of pressure in the systemic arteries. We refer to the bearing of this in cases of plethora.

Increase of the watery constituents of the blood increases the contraction volume and output to the same extent (though only temporarily) as does transfusion of blood, but acts more unfavourably on the heart, seeing that the work done by the ventricles is increased, while the nutritive value of the blood supplied to the coronaries is diminished.

The increased output of the heart both in plethora and in hydræmia is due to rise of pressure in the systemic veins increasing the volume of blood which enters the right ventricle during diastole. We refer to the bearing of these facts upon the treatment of chlorosis and heart disease.

We then consider the limits of the power of the heart to perform the work thrown upon it, and show that in strictly physiological conditions, and in spite of the beautiful mechanism by which the force of the ventricular contraction is regulated, the cardiac mechanism, like the other mechanisms of the body, is liable to derangement, inter alia from fatigua when the work thrown upon it greatly exceeds that which it usually has to perform. We take as an example the increased work thrown upon the organ during active muscular exertion, and show that exertion and enduraince of fatigue are limited mainly by the limited power of the heart to continue supplying the increased amount of blood which is required by the acting voluntary muscles. We find that those luxuries which are forbidden or limited in "training," and which are known to binder prolonged exertion, such as water, alcohol, tobacco, caffein, all directly' weaken the force of the heart's contractions, and, in the case of water, place the organ under further disadvantages; also, that fatigue of the heart leads to dilatation of the organ.

On comparing the power of fatigued ventricles to carry on increased work as compared with well-nourished, unfatigued ventricles, it is found that not only is the strengthening effect of the augmentor nerves upon the individual contractions less in the former case, but also that the fatigued and therefore dilated heart is per se unfavourably placed for meeting increase in the work thrown upon it. An explanation is given of the reason why in heart disease failure takes place during exertion.

The part played by the vagus in protecting the diseased heart from harmful overwork is referred to, and it is shown that irregularity of the heart in disease may be explained by the mode in which this nerve, when acting powerfully, releases the ventricles from the control of the rhythmic centre in the sinus. The chief forms of rhythmic and arrhythmic irregularity are considered, and it is shown that these correspond with the forms of irregularity which can be produced by vagus action. The irregular heart expends more energy, and its tissues, therefore, are more wasted, for a given amount of work than the heart which is beating regularly.

The effect upon the heart of imperfect aerration of the blood is, first of all, to produce powerful vagus action from the medullary vagus centre; this is usually, though not always, accompanied in curarised animals by diminution of the output of the heart. But reasons are given for assuming that the output would be increased in uncurarised animals, owing to the high venous pressure which results from struggling. Besides the vagus action, it can be shown that asphyxia causes. progressive weakening both of the auricles and of the ventricles, and attention is drawn to the fact that the considerable rise of pressure in the systemic arteries in asphyxia is accompanied by vagus effects upon the heart and not by augmentor action, as is the case, so far as we know, in all other instances in which the vaso-constrictor centre is excited in the normal individual.

It is noted that the changes in the heart and circulation which take place during asphyxia point to the conclusion that, when the total amount of oxygen in the blood is lowered, it is for the benefit of the economy that those organs, such as the central nervous system, whose continuous blood supply is a vital necessity, should be richly furnished with blood by constriction of the vessels of the spleen, kidney, and digestive system, whose blood supply can be cut off temporarily without danger to life, and also that the heart should carry on the circulation in a manner involving as little as possible waste of its own substance. This latter, as we have seen, it is the function of the vagus nerve to bring about.

\section{THE. NERVOUS ELEMENT IN MYXGEDEMA.}

BY JAMES R. WHITWELL, M.B.

[From the Laboratory of the West Riding Asylum, Menston, Leeds.]

IN the later part of the Report of the Myxœdema Committee of the Clinical Society of London, 1888, occurs the following pregnant passage: "Pathological observation, while showing cause for the changes in the skin observed during life, for the falling off of the hair and the loss of teeth, for the increased bulk of the body as due to the excess of the subcutaneous fat, affords no explanation of the affections of speech, movements, sensation, consciousness, and intellect, which form a large part of the symptoms of the disease"-in fact, the nervous element.

In myxœdema, then, there are recognised on all sides a certain series of symptoms which apparently are inexplicable, except on belief that they are due to some interference with the nervous apparatus, the most obvious being those enumerated. In explanation of these symptoms, two distinct views have been adopted by various authorities: (1) that all these nervous manifestations in myxœdema may be explained by an assumed coarse interference with afferent nerve conduction, owing to the demonstrable changes in the connective tissue throughout the body, this causing a secondary compli- 